\title{
IPHI, THE SACLAY HIGH-INTENSITY PROTON INJECTOR PROJECT
}

\author{
J-M. LAGNIEL \\ Commissariat à l'Energie Atomique - DSM - GECA \\ CEA-Saclay - LNS, 91191 Gif-sur-Yvette Cedex, France \\ S. JOLY, J-L. LEMAIRE \\ Commissariat à l'Energie Atomique - DAM - DRIF - DPTA - SP2A \\ B.P. 12 - 91680 Bruyères-le-Châtel, France \\ A. C. MUELLER \\ Centre National de la Recherche Scientifique - IN2P3 - IPN/Orsay \\ 91406 Orsay Cedex, France
}

\section{Abstract}

High-power accelerators are being studied for several projects based on high-flux neutron sources driven by proton or deuteron beams. Since the front end is the most critical part of such accelerators, it has been decided to build a High-Intensity Proton Injector (IPHI) designed to accelerate a cw $100 \mathrm{~mA}$ beam up to $11 \mathrm{MeV}$. The aim is to validate design codes, test technical choices, demonstrate operational reliability and measure the beam parameters in order to optimize the high-energy part of the accelerator. The design chosen for IPHI (ECR source, RFQ, DTL and diagnostic line) and the construction schedule are presented.

\section{INTRODUCTION}

Accelerators for high beam power (>1 MW) are studied for several applications such as fundamental research, accelerator-driven transmutation technologies (transmutation of radioactive wastes, tritium production), material science, energy production (hybrid reactors and fusion)... [1] [2]. For most of these applications, highpower proton or deuteron beams are used to produce high neutron fluxes with characteristics which cannot be obtained from nuclear reactors. One of the main advantages of an accelerator-driven neutron source is its flexibility : the neutron flux can be continuous or pulsed with time characteristics defined by users and the energy of the neutrons can be adjusted ( $14 \mathrm{MeV}$ neutrons for the fusion material tests). In addition, problems associated with reactors such as potential impact on the environment, supply of highly-enriched uranium, cost associated with spent fuel processing and decommissioning can be avoided or greatly minimized. However, this new generation of accelerators has to produce multi-MW beams. This is one to two orders of magnitude higher than the most powerful machines in operation today.

Careful attention must be paid to optimize the design of such high-power accelerators. On one hand, one needs to provide desired beam with high reliability for the operational life cycle. On the other hand, the capital and operational costs need minimization. The two main issues in this regard are the requirements of extremely low beam loss [3] and high availability. The final design results from a compromise between the technical risk and the total cost of the project. A good compromise is difficult to achieve without a serious $R \& D$ program focused on the important issues mentioned above. Since rf linacs have emerged as the accelerators of choice for pulsed or $\mathrm{cw}$ beams above $5 \mathrm{MW}$, we have undertaken a comprehensive demonstration program for the low-energy part of such machines.

\section{IPHI R\&D PROGRAM AIMS}

IPHI is a R\&D project proposed by the CEA with collaborations from CNRS. The project is also supported by Thomson-CSF-AIRSYS, a company with experience in the construction of accelerators for medicine, industrial and military applications. The project aim is to build a $11 \mathrm{MeV}$ "Injector for Protons with High Intensity" (> $100 \mathrm{~mA}$ ) capable of operation with duty cycle up to $100 \%$ (see section III). The goal of the R\&D program is to gain knowledge necessary to optimize both design and cost of several projects of interest to the IPHI partners :

- TRISPAL, the French project for tritium production [2],

- IFMIF, the International Fusion Materials Irradiation Facility [4],

- ESS, the European Spallation neutron Source [5],

- nuclear waste transmutation and next generation of exotic beam facilities.

After an analysis of the best $R \& D$ program to undertake which would serve all these projects, a proposal to build the IPHI prototype has been made. The same has been done in Canada (the Chalk River Injector Test Stand is presently undergoing tests at Los Alamos [6]), in Japan (JAERI Basic Test Accelerator [7]) and in USA at Los Alamos (Low-Energy Demonstration Accelerator [8]). Each of these high-power accelerator front ends (under test or in progress) has different designs and would therefore provide complementary knowledge bases for optimization of both design parameters and costs of this new class of high-power accelerators. They specifically will allow :

- validation of the beam dynamics codes by testing them in the low-energy sections where space-charge effects are dominant, 
- knowledge of the beam distribution at an energy where halo considerations become crucial $(\sim 10 \mathrm{MeV})$,

- demonstration of a specific set of technological choices and adequacy of design codes (RF cavities, RF systems, temperature control systems, diagnostics for high-power beams,...),

- accumulation of data on reliability, availability and maintainability, cost of the accelerator components and ability of the manufacturers to build them.

It is clear that all that information gained at low energy is essential to optimize a high-energy machine.

\section{LAYOUT}

The High-Intensity Light-Ion Source SILHI is the first stage of the IPHI prototype. It has been designed to produce high-intensity proton or deuteron beams (> $100 \mathrm{~mA}$ ) at $95 \mathrm{kV}$ with up to $100 \%$ duty cycle. Design started in mid-1994 with funding from CEA, and teams from CEA-DSM (Saclay and Grenoble) and 'Laboratoire National Saturne' (CEA-DSM and CNRS-IN2P3). A
$2.45 \mathrm{GHz}$ ECR source was chosen in light of the excellent performances achieved at Chalk River and Los Alamos. This type of source has also several advantages compared to the other source types :

- no filament or RF antenna,

- low microwave power $(<1 \mathrm{~kW})$,

- very high gas efficiency,

- good beam stability,

- high proton or deuteron fraction (> 90\%).

Within two weeks of the end of the construction (July 1996), a $46 \mathrm{~mA} \mathrm{cw}$ total beam current was extracted at $70 \mathrm{kV}$ using a $\phi=10 \mathrm{~mm}$ aperture plasma electrode (plasma density: $\mathrm{J}=58 \mathrm{~mA} / \mathrm{cm}^{2}$ ). Six months later, a $107 \mathrm{~mA} \mathrm{cw}$ beam current was obtained at the nominal extraction voltage $(95 \mathrm{kV})$ with a $\phi=8 \mathrm{~mm}$ aperture plasma electrode $\left(\mathrm{J}=213 \mathrm{~mA} / \mathrm{cm}^{2}\right)$. Continuous beam above $90 \mathrm{~mA}$ are now accelerated routinely. Measured beam characteristics are reported in Ref. 9.

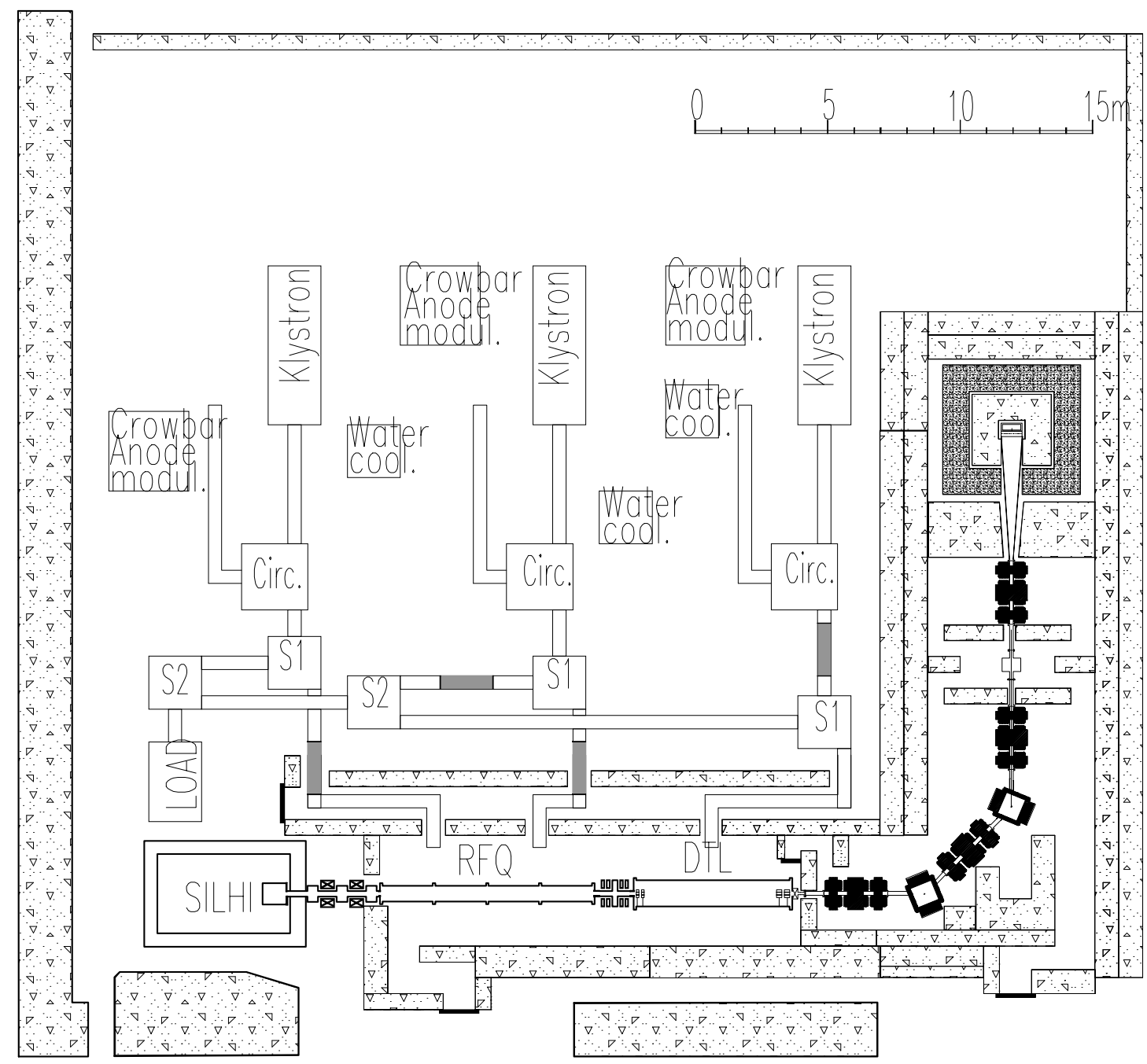

Figure 1: Layout of the IPHI prototype 
The next steps of the IPHI project concern the construction of a RFQ followed by a DTL tank in order to reach $11 \mathrm{MeV}$ (fig.1). The IPHI partners are ready to support both construction and manpower costs for this new stage since 1997.

The IPHI RFQ will be a $\sim 7 \mathrm{~m}$ long four-vane structure following the developments at the Los Alamos National Laboratory. As in the APT RFQ, the cavity will be brazed and will be made from several separately tunable segments assembled with RF couplings [8]. The IPHI RFQ is designed to work with two $352 \mathrm{MHz}$ highpower RF generators of the CERN (LEP) and ESRF type. For the present design, beam dynamics calculation shows a beam transmission of $97 \%$ for $100 \mathrm{~mA}$ with both multipoles and image-charge effects taken into account. Investigations are presently being done to improve this design (same transmission with a maximum electric field lower than 1.8 Kilpatrick criterion...). The final energy is $5 \mathrm{MeV}$, the lowest energy which allows to build the first drift tube of the $352 \mathrm{MHz}$ DTL using electromagnetic quadrupoles.

It is planned to build a Beam Matching Section between the IPHI RFQ an DTL in order to keep some freedom for the tunings and to leave enough room to install diagnostics.

The IPHI DTL will be a $6 \mathrm{~m}$ long Alvarez structure designed to accelerate beam to $11 \mathrm{MeV}$. The choice of a conventional DTL structure for IPHI is mainly done to avoid emittance growth and halo formation which can be induced when the energy gain per focusing period is high. A $2 \pi$ mode DTL structure with a FODO focusing period leads to the shortest focusing period $(2 \beta \lambda)$ with the best ratio for the linear radial focusing strength (quadrupoles) over the nonlinear RF defocusing effect. Also, many DTL Alvarez structures have successfully demonstrated their capabilities to accelerate very high pulsed beam currents (200 mA and above) worldwide. The main issue for a high duty-cycle DTL is to cool the low-energy drift tubes which house high-gradient quadrupoles. Studies undertaken for the IPHI project indicate that the drift tubes can be adequately cooled for the $352 \mathrm{MHz} \mathrm{cw}$ structure above $5 \mathrm{MeV}$. The first task on the IPHI DTL will be to build a hot prototype with a small number of drift tubes ( 2 or 3 ) for feasibility demonstration.

The IPHI Diagnostic Line being studied will provide both transverse and longitudinal emittance measurements for the RFQ beam (up to $500 \mathrm{~kW}$ ) and subsequently for the full DTL beam (up to $1 \mathrm{MW}$ ). It is planned to vary the focusing and energy dispersion properties of this line to perform these measurements using non-destructive profile monitors. The beam halo will be measured on collimators located all along the line.

\section{CONCLUSION}

The final decision for the IPHI project is expected by June 1997. A favorable decision will allow to start this
R\&D program for the future generation of high-power linear accelerators. This will be mainly funded by CEA with a total investment budget of $90 \mathrm{MFF}(\sim \mathrm{M} \$ 18)$. The total manpower need is estimated to be 240 man-years for the entire duration (1997-2002) of the project with 50 man-years per year during the construction period (19982000).

The IPHI prototype will be installed in the CEASaclay Center. The key dates are :

$$
\begin{aligned}
& \text { - 06/2000 : first RFQ beam } \\
& \text { - 06/2001 : end of the RFQ tests ( } 500 \mathrm{~kW} \text { beam) } \\
& \text { - 09/2001 : first DTL beam } \\
& \text { - 12/2002 : end of the program ( } 1 \mathrm{MW} \text { beam) }
\end{aligned}
$$

\section{ACKNOWLEDGEMENT}

The authors gratefully acknowledge the SILHI and IPHI Team members for the large amount of excellent work already done. The contributions of C. Perraudin and C. Bourat have been also highly appreciated. They sincerely thank all the authorities who support the project: M. Olivier, J. Faure, S. Harar and C. Césarsky for CEA-DSM; J. Daussy, A. Joubert and J. Bouchard for CEA-DAM; S. Galès, F. Dupont and C. Détraz for CNRSIN2P3; J. Courau for Thomson-CSF-AIRSYS. Many thanks also to our colleagues and friends from LANL, JAERI and IFMIF Team for fruitful discussions with special thanks to S. Nath.

\section{REFERENCE}

[1] S.O. Schriber, "Survey of proposed high intensity accelerators and their applications", EPAC94 Proc., June 1994, Vol.1 p.213.

[2] M. Promé, "Major projects for the use of high power linacs", LINAC96 Proc., August 1996, Vol.1 p.9.

[3] J-M. Lagniel, "Halos and chaos in space-charge dominated beams", EPAC96 Proc., June 1996, Vol.1 p.163.

[4] IFMIF CDA Team, "International Fusion Materials Irradiation Facility Conceptual Design Activity, Final Report", Edited by M. Martone, ENEA-Frascati, Italy, December 1996, RT/ERG/FUS/96/11.

[5] "Outline design of the European Spallation Source", Edited by I.S.K Gardner, H. Lengeler and G.H. Rees, September 1995, ESS report 95-30-M.

[6] J.D. Schneider et al., "Installation of a cw radiofrequency quadrupole accelerator at Los Alamos National Laboratory", LINAC94 Proc., August 1994, Vol.1 p.149.

[7] K. Hasegawa et al., "The R\&D status on the front end of the high intensity proton accelerator in JAERI", LINAC96 Proc., August 1996, Vol.2 p.665.

[8] J.D. Schneider, "APT accelerator technology", LINAC96 Proc., August 1996, Vol.1 p.22.

[9] P-Y. Beauvais al., "First beams of the CEA-Saclay cw high-intensity microwave source", PAC97, 6W \#5347. 